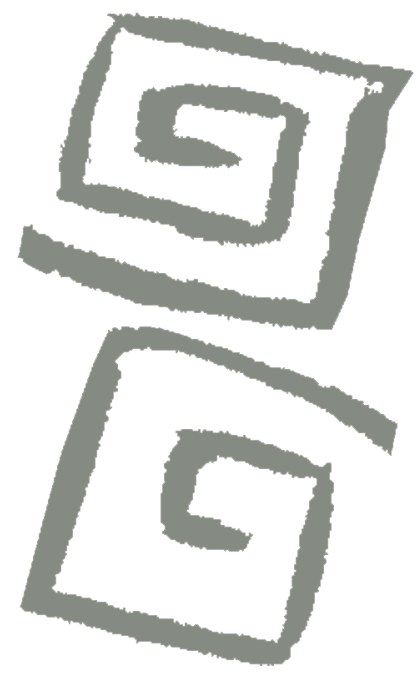

\title{
La emergencia de la diabetes en una comunidad tapiete de Salta: género, etnicidad y relaciones con el sistema de salud
}

\author{
The emergence of diabetes in a Tapiete community of \\ Salta: gender, ethnicity and relations with the health \\ system
}

Silvia Hirsch' ${ }^{1}$, Valeria Alonso ${ }^{2}$

'Autora de correspondencia. Doctora en Antropología. Investigadora, Instituto de Altos Estudios Sociales, Universidad Nacional de San Martín, San Martín, Buenos Aires, Argentina. $\bowtie$ (iD

${ }^{2}$ Magíster en Ciencias Sociales y Salud. Investigadora, Instituto Nacional de Epidemiología, Administración Nacional de Laboratorios e Institutos de Salud, Mar del Plata, Buenos Aires, Argentina. $\triangle$ (iD)
RESUMEN En Argentina, la ocurrencia de diabetes y otras enfermedades crónicas en población indígena aún no ha sido suficientemente estudiada. Este trabajo es resultado de una exploración etnográfica de las determinaciones socioculturales y de género de los procesos de atención y prevención de diabetes en indígenas tapietes de la provincia de Salta. La investigación combinó observación participante con entrevistas a varones y mujeres de edad adulta y con diagnóstico de diabetes, y entrevistas a enfermeras y médicas de un centro de atención primaria de Misión Los Tapietes y de un hospital de Tartagal, entre los meses de agosto y septiembre de 2019. En la comunidad se ha observado la creciente presencia de diabetes, junto con la falta de articulación de las intervenciones sociosanitarias con las prácticas nativas de salud-enfermedad-atención; los problemas en la comunicación del diagnóstico y tratamiento; y las dificultades de acceso al sistema de salud. El artículo discute la complejidad de la transición epidemiológica, los sentidos de la enfermedad crónica para los varones y las mujeres, la emergencia traumática de la diabetes y las dificultades en el ejercicio del derecho a la salud de los pueblos originarios.

PALABRAS CLAVES Género y Salud; Diabetes Mellitus; Salud de Poblaciones Indígenas; Argentina.

\begin{abstract}
In Argentina, the presence of diabetes and other chronic diseases among indigenous populations has not yet been sufficiently studied. This article is the result of an ethnographic study of the sociocultural and gender determinants that are involved in diabetes care and prevention processes among the Tapiete people in the province of Salta. The research combined participant observation and interviews both with adult men and women diagnosed with diabetes and with nurses and doctors at a primary care center in Misión Los Tapietes and at a hospital in Tartagal between August and September of 2019. The growing presence of diabetes has been observed in the community, along with a lack of articulation of social and health interventions with native practices of healthdisease-care, as well as problems in communicating diagnosis and treatment options, intensified by difficulties regarding access to the healthcare system. The article examines the complexity of the epidemiological transition, meanings of chronic disease for men and women, the traumatic emergence of diabetes, and difficulties in exercising the right to health among indigenous populations.
\end{abstract}

KEY WORDS Gender and Health; Diabetes Mellitus; Health of Indigenous Populations; Argentina. 


\section{INTRODUCCIÓN}

Una tarde en Tartagal conversando con R, un tapiete de Salta, sobre su padecimiento de diabetes, nos dijo que a la gente no le gusta hablar de esta enfermedad porque "es algo feo, algo que avergüenza". La diabetes no solo avergüenza, es invisibilizada, resistida y negada. En Argentina, la ocurrencia de diabetes y otras enfermedades crónicas en población indígena aún no ha sido suficientemente estudiada en sus múltiples dimensiones socioculturales, políticas, económicas y sanitarias. Este trabajo es resultado de una exploración etnográfica de las determinaciones socioculturales y de género que intervienen en los procesos de salud, enfermedad, atención y prevención de la diabetes en indígenas tapietes de la provincia de Salta y su articulación con los efectores de salud pública. Con el propósito de comprender las dificultades en el acceso al sistema de salud y la relación con la biomedicina, la exploración tuvo como objetivos identificar diferenciales en los itinerarios terapéuticos de varones y mujeres con diabetes y describir su articulación con los efectores de salud pública. Forma parte del proyecto "Estudio socio-antropológico de enfermedades crónicas no transmisibles en comunidades guaraníes y tapietes de la provincia de Salta", respaldado por el Instituto de Altos Estudios Sociales de la Universidad Nacional de San Martín y el Instituto Nacional de Epidemiología de la Administración Nacional de Laboratorios e Institutos de Salud.

La incorporación de la perspectiva de género al estudio de la diabetes y otras enfermedades crónicas en las comunidades permite contextualizar la vulnerabilidad diferencial entre varones y mujeres, los modos de vivir, enfermar y morir, según perfiles epidemiológicos de género. A su vez, la consideración de la variable étnica, generalmente ausente en los registros epidemiológicos, responde a un área de vacancia para el desarrollo de una epidemiología sociocultural ${ }^{(1)}$. La diabetes es un problema epidemiológico de actualidad, recientemente abordado por los estudios de género. Estudios cualitativos encontraron que las diferencias de género inciden en la significación de la diabetes con impacto en las prácticas de cuidado $^{(2)}$ y se hallaron desventajas para el autocuidado en las mujeres y mayor vulnerabilidad para afrontar el control glucémico en los sectores populares ${ }^{(3,4)}$. Estas desventajas se asociaron con déficit en el manejo de los recursos económicos, en el acceso a la información y en la posición subalterna de las mujeres en la estructura doméstica ${ }^{(5)}$. La incorporación de la perspectiva de género y de etnicidad al estudio de la diabetes y otras enfermedades crónicas en las Comunidades indígenas permite contextualizar la vulnerabilidad diferencial entre varones y mujeres, los modos de vivir, enfermar y morir, según perfiles epidemiológicos de género y etnicidad.

En las comunidades indígenas se ha observado la creciente presencia de obesidad, diabetes e hipertensión, junto con la falta de articulación de las intervenciones sociosanitarias con las conceptualizaciones y prácticas nativas de salud-enfermedad-curación, los problemas en la comunicación del diagnóstico y tratamiento y las dificultades de acceso al sistema público de salud. La diabetes tipo 2 se asocia a una serie de cambios en la alimentación y la forma de vida (una dieta rica en azúcar, grasas y poca o nula actividad física), y a factores genéticos. La aparición de este tipo de diabetes está ligada a formas de vida occidentales, en las que la determinación social es central a la etiología y al tratamiento de esta condición de salud ${ }^{(6)}$. En la población indígena, la frecuencia de diabetes también ha aumentado en las últimas décadas y los factores que explican la presencia de esta enfermedad son múltiples: cambios en la alimentación, sedentarismo, factores genéticos y otros. Ferreira y Lang $^{(7)}$ consideran la diabetes como una patología con una impronta sociopolítica y proponen analizar esta enfermedad más allá del cuerpo del sufriente e indagar en las consecuencias que ha tenido el colonialismo sobre los pueblos indígenas y el impacto en sus cuerpos y fisiologías. Esta enfermedad crónica genera consecuencias adversas no solo en la salud de quienes la 
padecen, sino también en sus familias y en sus comunidades. El abordaje que damos a la diabetes toma en cuenta los factores históricos, sociales y económicos que afectaron a la comunidad tapiete, en la que se produce una situación traumática, considerada como un estado de pérdida no resuelta, que se transmite generacionalmente y que incide sobre los cuerpos y las subjetividades de las personas. Este trabajo intenta articular teoría, historia y etnografía en la comprensión del proceso de salud-enfermedad-atención y prevención del padecimiento crónico en perspectiva de género, de manera de abrir futuras líneas de investigación en antropología de la salud y epidemiología sociocultural.

\section{Breve historia de los tapietes}

Los tapietes son un grupo originario del Chaco boliviano, que atravesó profundos e intensos cambios a partir de la relación con la sociedad nacional, de sus procesos migratorios y su asentamiento permanente en la comunidad "Misión los Tapietes" ubicada en la ciudad de Tartagal, provincia de Salta. El pueblo tapiete comenzó a migrar a principios del siglo XX al norte argentino, para trabajar en los ingenios azucareros y como consecuencia de la Guerra del Chaco que tuvo lugar entre 1932 y 1935 entre Bolivia y Paraguay. En los ingenios azucareros y fincas donde trabajaron junto a muchos otros grupos indígenas chaqueños, conocieron y les fueron impuestas nuevas formas de alimentación. En los ingenios había mujeres conocidas como "tacheras" que preparaban comida para los trabajadores. Guisos, puchero, mate cocido con azúcar y pan fueron los alimentos más consumidos. Estas prácticas alimentarias, sumadas al posterior consumo de mate cebado, bebidas gaseosas y alcohol implican un elevado consumo de azúcar. A partir de su asentamiento en Tartagal se redujeron las prácticas de caza y recolección; no obstante, en la zona aledaña a su asentamiento cultivaban chacras con mandioca, maíz, zapallo, y hortalizas y criaban gallinas. Pero en la década de 1970, el municipio tomó la decisión de lotear el asentamiento de la comunidad: se eliminaron las chacras y se redujo el espacio a lotes de 20×15 metros para las familias. Esta reducción del espacio produjo una dependencia absoluta de la compra de alimentos, y la incorporación cada vez mayor de alimentos industrializados.

La incorporación de estos alimentos se relaciona directamente con la sociedad circundante, con la escolarización y el trabajo fuera de la comunidad que transformaron las prácticas culturales cotidianas. En las décadas de 1950 y 1960 aumentó el consumo de alcohol, particularmente entre los hombres. Aparejado a estos cambios y a la pérdida del espacio para vivir y cultivar, se añade la retracción del uso de la lengua materna en el proceso de escolarización y frente a la sociedad circundante. A partir de las décadas de 1960 y 1970 ingresa el evangelio, que llevó a la conversión de prácticamente todo el grupo, y que también va a incidir en la forma de vida cotidiana, la alimentación y en la reducción del consumo del alcohol. Si bien las transformaciones culturales y sociales que atravesó la comunidad implicaron nuevas formas de vida, como el sedentarismo, el abandono de formas de subsistencia y de prácticas ancestrales, persisten en la comunidad conocimientos en torno al uso de plantas medicinales y tratamientos provenientes de sus propia farmacopea ${ }^{(8)}$.

\section{Abordajes teóricos: transformaciones socioculturales y trauma histórico y colectivo}

Algunos autores refieren a la noción de trauma colectivo y trauma histórico que enfrentan muchos pueblos originarios, y que sirve para entender los síntomas de pérdida histórica que se manifiestan en la depresión, el alcoholismo, la diabetes, el desempleo, entre otros. Brave Heart y Debruyn ${ }^{(9)}$ utilizaron el concepto de trauma histórico tomado de la bibliografía sobre el holocausto. Estos autores indican que muchos de los problemas que enfrentan los indígenas en EEUU son el resultado de un legado de trauma 
crónico, de pérdida no resuelta que se transmite generacionalmente. Un aspecto distintivo del trauma histórico es que se transfiere a las siguientes generaciones a través de medios biológicos, psicológicos, ambientales y sociales. La pérdida de tierras implicó la falta de acceso al hábitat como lugar para procurar alimentos, como espacio sagrado, lo cual condujo al concomitante proceso de urbanización. A esto se suma el proceso civilizatorio caracterizado por la evangelización, el trabajo asalariado y la escolaridad. Todos estos factores y otros generaron la disrupción de la estructura económica, de los vínculos familiares, de las prácticas sociales y rituales, y el uso de la lengua.

Las experiencias traumáticas causan estrés e irrumpen en el cuerpo a través de diversas sintomatologías: enfermedades cardiovasculares, diabetes, problemas gastrointestinales, cáncer, a las que se suman los problemas de salud vinculados a determinados trabajos asalariados (traumatológicos y musculares), tales como hachar, lavar ropa, cosechar. Korn y Ryser ${ }^{(10)}$ indican que la diabetes, los problemas cardíacos y la obesidad forman parte del trío del trauma comunal que es el resultado del desarrollo inducido. El trauma colectivo está "constituido por los eventos que apabullan las capacidades de la comunidad para funcionar de maneras estables y generativas" ${ }^{\prime 111}$. La destrucción del medio ambiente, la transformación económica, la interrupción de la seguridad alimentaria, la disrupción del orden social, la relocalización física, la colonización educativa y la conversión religiosa son características del desarroIlo externamente definido y que conducen al trauma comunitario.

Esto, a su vez, se vincula con un trauma nutricional y con una dramática transición alimentaria, por la cual los alimentos que se consumen ya no son producidos por la gente, sino que son adquiridos. Los alimentos tradicionales requieren mayor tiempo de preparación y, en el norte de país, constituyen un diacrítico de lo indígena, de una etnicidad subalternizada y excluida, y no son consumidos por los vecinos criollos. Lo que indican estos autores es que el estrés es un detonante de la hiperglucemia y el desarrollo de la diabetes, y este estrés se combina con el trauma nutricional que afecta a los pueblos indígenas. En el proceso de salud-enfermedad-atenciónprevención, los padecimientos o daños a la salud son parte de un proceso social dentro del cual se va formando colectivamente la subjetividad. Desde un enfoque relacional, se reconoce que los sujetos y grupos sociales asumen estrategias de prevención que combinan saberes médicos tradicionales, populares y científicos $^{(12)}$. Desde una concepción que entiende la enfermedad como hecho social, propia de la antropología de la salud, se entiende la estrategia de vida en su asociación a las trayectorias de los padecientes en los contextos sociales que les dan soporte ${ }^{(13)}$. De modo que el padecimiento y la enfermedad no solo constituyen hechos biológicos, sino también son construcciones sociales, con significaciones y sentidos que impactan en los procesos de recuperación de la salud y la eficacia terapéutica de los tratamientos ${ }^{(14)}$. Los itinerarios terapéuticos son definidos como las trayectorias de los pacientes entre distintos modelos de atención del padecimiento en contextos de pluralismo médico ${ }^{(15)}$. Las prácticas alimentarias se comprenden en tanto relación social que incluye la organización de su producción, distribución y consumo, así como las creencias religiosas, sanitarias y gastronómicas. Se entiende que, en la emergencia de las enfermedades crónicas no transmisibles como obesidad, hipertensión, hipercolesterolemia, diabetes mellitus, cáncer, y accidentes cardio y cerebrovasculares, han jugado un rol protagónico los alimentos industrializados ultraprocesados que contienen gran parte de grasa saturada, azúcar (sacarosa) refinada, jarabe de alta fructosa, sal y aditivos ${ }^{(16)}$.

\section{METODOLOGÍA}

El estudio se basa en los criterios de la investigación cualitativa con un diseño descriptivo de enfoque etnográfico. Desde los aportes teóricos y metodológicos de la antropología de la salud y la epidemiología sociocultural, 
entendemos que las etnografías posibilitan construir el perfil epidemiológico de un grupo determinado, incluyendo información económica, política y sociocultural. Con estas aproximaciones podemos encontrar información respecto de enfermedades generalmente no diagnosticadas o aspectos de ellas no considerados por la investigación epidemiológica clásica, y obtener sus interpretaciones con base en la perspectiva de los actores, que permiten diseñar actividades específicas ${ }^{(17,18)}$.

El estudio de la presencia de diabetes en la comunidad comenzó en el año 2016; sin embargo, entre los meses de agosto y septiembre de 2019 realizamos trabajo de campo de manera más sistemática en Misión Los Tapietes, en Tartagal. Asimismo, la investigación previa realizada en la comunidad, desde 2002, por Silvia Hirsch, permitió tener una perspectiva longitudinal por la cual se registró la creciente presencia de diabetes, en particular, en la última década. La investigación combinó la observación participante con la realización de entrevistas semiestructuradas y abiertas a varones y mujeres de edad adulta con diagnóstico de diabetes y enfermeras y médicas del Centro de Atención Primaria de la Salud (CAPS) de Misión Los Tapietes y del Hospital "Juan Domingo Perón" de Tartagal.

La población de esta primera etapa del estudio estuvo conformada por miembros de la comunidad tapiete. Los criterios de inclusión contemplaron varones y mujeres mayores de 18 años, sin límite superior de edad, residentes en la comunidad, que hubieran recibido un diagnóstico de diabetes de un efector de salud.

Misión Los Tapietes es una comunidad periurbana localizada a diez cuadras del centro y del hospital de la ciudad de Tartagal. La población de la Misión es de aproximadamente 850 personas según datos del Programa de Atención Primaria de la Salud de la provincia de Salta, en Tartagal. En la actualidad, la comunidad ocupa cinco manzanas. Cuenta con un CAPS equipado con consultorios, atendido por una enfermera, una médica clínica, un médico obstetra, una nutricionista y una administrativa.
La muestra cualitativa, de tipo intencional, para la realización de entrevistas incluyó a nueve pacientes con diabetes (cuatro varones y cinco mujeres) y cuatro profesionales: una enfermera y una médica del CAPS, y dos médicas del hospital de Tartagal (una médica familiar y una diabetóloga recientemente retirada de la salud pública).

Para la población indígena identificada con diagnóstico de diabetes se elaboraron pautas de entrevista orientadas a conocer los aspectos más relevantes de la enfermedad, los itinerarios terapéuticos, las dificultades en los tratamientos, las complicaciones y el acceso a los servicios de salud. Se realizaron entrevistas semiestructuradas a las profesionales de salud: se indagó en los conocimientos que poseen sobre el curso de los pacientes con diabetes y en las propias percepciones acerca de la eficacia de los dispositivos de seguimiento de los pacientes. Se realizó observación participante en la comunidad, el CAPS y el hospital.

Para el análisis de la información se transcribieron las entrevistas y sistematizaron las observaciones: los datos se organizaron en torno a los ejes temáticos de causas, síntomas, tratamiento, control, seguimiento, complicaciones, relación con el sistema de salud y otros sistemas de atención de la salud disponibles ${ }^{(19)}$. La información se analizó en tres niveles: macro, programas de salud y alimentación dirigidos a la población indígena; meso, efectores públicos de salud a nivel local, CAPS, prácticas locales de alimentación; y micro, atención de salud y relación con los pacientes, las prácticas terapéuticas de los pacientes y el acceso a los programas nutricionales.

De acuerdo con una perspectiva de género que aporta una mirada hacia las particularidades de las prácticas de cuidado y atención diferencial entre los varones y las mujeres, los resultados de las entrevistas se agruparon en las siguientes dimensiones: las circunstancias del diagnóstico, las características del tratamiento, las causas atribuidas a la diabetes, formas de nombrar la diabetes, el imaginario de la enfermedad crónica, y la manera de dar sentido a la solución del problema de la diabetes. La información de las 
entrevistas a profesionales se clasificó según la caracterización de la perspectiva de salud intercultural, la evaluación del impacto de la diabetes, las particularidades del tratamiento y el seguimiento de las y los pacientes y su acceso a los servicios de salud pública, la apreciación de los resultados de salud y la cobertura de programas nutricionales. Con el fin de preservar la confidencialidad, los nombres de las y los participantes fueron reemplazados por iniciales.

El estudio cumple de manera interrelacionada y complementada con los principios de dignidad humana y derechos humanos, respeto de la vulnerabilidad humana y la integridad personal, privacidad y confidencialidad, igualdad, justicia y equidad, no discriminación y no estigmatización, respeto de la diversidad cultural y del pluralismo, solidaridad y cooperación, responsabilidad social y salud, aprovechamiento compartido de los beneficios, protección de las generaciones futuras, protección del medio ambiente, la biósfera y la biodiversidad de la Declaración Universal Sobre Bioética y Derechos Humanos de la Unesco, del año 2005. El proyecto fue aprobado por el Comité de Ética en Investigación del Instituto Nacional de Epidemiología (código: Alonso/Hirsch-01/2018) y, de acuerdo con la Resolución 1480/2011 del Ministerio de Salud, se solicitó el consentimiento informado a las y los participantes de la investigación y a las profesionales de la salud que fueron entrevistaron en el CAPS y el hospital.

\section{LA EXPERIENCIA DE LA DIABETES}

Las personas padecientes de diabetes se enteran de la enfermedad, generalmente, cuando ya tienen síntomas muy avanzados: aumento de la micción, mareos, desmayos, infecciones recurrentes, visión borrosa. Cuando reciben el diagnóstico de diabetes y la indicación de los cuidados, los pacientes parecen realizar distintas interpretaciones que se alejan de las descripciones biomédicas de la enfermedad. En la comunidad tapiete, no se conoce la noción de cronicidad, de hecho en la lengua tapiete, no hay una palabra para traducir el significante crónica o explicar que una enfermedad puede ser de por vida. La mayoría de los entrevistados respondió que no conocía el término "enfermedad crónica" y que tampoco recibieron explicaciones al respecto. $\mathrm{Al}$ preguntar a L si había escuchado hablar de enfermedad crónica, respondió: "No, nunca escuché" y M expresó: "Quisiera saberlo, no entiendo la palabra".

Esta cualidad del significado en sí misma incide precisamente en la continuidad del tratamiento y en la comprensión de las consecuencias de la diabetes mellitus. Al respecto, la señora F explica: "El Dr. [T] me dijo 'vos sabías que te sentías mal, y no te vas a asustar. Vos sabés que no te vas a curar'", a lo cual la señora respondió "hay muchos remedios, igual va a terminar mi cuerpo cuando el Señor diga".

Así, entre la evitación y la resistencia, los tratamientos para la diabetes se siguen de manera discontinua, se abandonan, se reemplazan por prácticas y creencias religiosas. Para los padecientes de diabetes, esta enfermedad oscila entre momentos en que se sienten bien, aumentan de peso, disminuyen los síntomas, y momentos de desmejora que, con frecuencia, conducen a una internación. En este sentido, cabe indicar que no suelen adherir de manera cotidiana a las restricciones alimentarias propias del tratamiento de la diabetes. Se recurre a la dieta, por lo general, cuando se acude al hospital por situaciones de gravedad.

Los tapietes con diabetes llegan descompensados al hospital de Tartagal, con pérdida drástica de peso, deshidratación y problemas renales; siguen los tratamientos de manera intermitente, comienzan a reducir el consumo de azúcar y grasas y, en algunos casos, recurren a los medicamentos indicados por un tiempo. Incorporan el edulcorante para tomar mate, pero el mate siempre es acompañado por pan. Cuando comienzan a sentirse mejor, retornan a las prácticas alimentarias habituales y abandonan la dieta o las restricciones alimentarias. Muchos pacientes con diabetes recurren a hierbas medicinales para tratar la diabetes, pero solo en algunos 
casos las combinan con medicamentos recetados. Tanto hombres como mujeres consumen medicamentos tales como metformina y glibenclamida de forma irregular, pero usan con más frecuencia plantas medicinales, tales como la altamisa, la moringa, la limonada amarga y la uña de gato. En ambos casos, se recurre a la oración y la sanación espiritual en la iglesia evangélica, con algunas restricciones en la dieta. Según indicó I, una mujer de 55 años:

...uso medicamentos, y plantas como la hoja de guayaba en té, y voy a la casa de una señora tapiete que hace curas. A las plantas las busco en la comunidad chorote, mi hermana vive ahí. Ellos me oran, me visitan en su casa. Yo les dije que no podía respirar, ellos me oran y yo me siento mejor. (I, mujer, 55 años)

Tres de las mujeres entrevistadas, presentan la doble condición de diabetes más tuberculosis o Chagas: el tratamiento medicamentoso de tuberculosis parece extenderse al tratamiento de la diabetes. Mientras se cura la tuberculosis, abandona el tratamiento de la diabetes. La señora F, indicó:

....ahora estoy dejando la pastilla [refiriéndose a la metformina] y estoy tomando remedios caseros: hoja de mango hervida, hoja de papaya machacada muy amarga, uña de gato, no voy al centro de salud. $(F$, mujer, 65 años)

A doña $L$, de 62 años, le detectaron Chagas hace poco tiempo atrás, y diabetes hace ocho años. Nos contó que cuando le dieron el diagnóstico se asustó, además tiene la presión alta, y cuando tiene el azúcar alta se siente afligida.

En las prácticas cotidianas de cuidado $y$, en particular, en la preparación de alimentos, hemos observado las diferencias de género más significativas en cuanto a la experiencia de la diabetes. Las mujeres son quienes preparan la comida, y esto constituye un desafío cotidiano, dado que deben articular las restricciones en la dieta indicada por la diabetes con los deseos y prácticas de alimentación del grupo familiar. Al haber perdido el acceso a las tierras para cultivo y el espacio para caza y recolección, viven en un contexto de total dependencia a los alimentos comprados y de transformación de las prácticas de alimentación, lo cual torna sumamente difícil preparar varias comidas para los diferentes miembros de la familia. En este sentido, si bien recae sobre las mujeres las prácticas de cuidado del padeciente de la diabetes y de sí misma -lo que implica la preparación de determinada dieta-, en un contexto de carencia, formado por grupos familiares compuestos por diversidad etaria, esto restringe la adhesión a tratamientos indicados por los efectores de salud. De hecho, en las entrevistas es recurrente la mención a la dificultad de seguir la dieta prescripta. El consumo de gaseosas azucaradas, mate con azúcar y pan, son parte no solo de las prácticas alimenticias, sino también de la sociabilidad y comensalidad. Los relatos de varones y mujeres vinculan frecuentemente la diabetes con la comida, especialmente, con el consumo en exceso de carne y bebidas gaseosas azucaradas, que resalta una dimensión histórica de pérdida de prácticas de producción y preparación de los alimentos: "ahora nadie siembra", "antes se cocinaba hervido o al rescoldo" mencionaron varios entrevistados.

La diabetes también es referida como consecuencia del estrés emocional. En este sentido, una de las señoras entrevistadas se refirió al impacto que tuvo en su vida la muerte de su hija de 9 años:

\section{...no podía comer, escuchaba la voz de mi hija en el oído: mami tengo hambre y yo no podía comer más. ( $\mathrm{F}$, mujer, 65 años)}

Otras mujeres refirieron nervios y el consumo de "cosas dulces" como posibles determinantes de la irrupción de la diabetes en su vida. Pero, también el dolor por la pérdida de un hijo fue mencionado por $\mathrm{R}$, un hombre de 60 años, quien relató que después de la muerte de su hijo por cáncer, enfermó de diabetes.

Si bien la mayoría de las personas entrevistadas utilizaron el término diabetes, en la 
lengua tapiete, a la diabetes se la denomina "sangre dulce". De manera congruente con el desconocimiento de la enfermedad crónica, tanto varones como mujeres coinciden en las perspectivas de curación de la enfermedad: se articulan la confianza en Dios, en los remedios naturales y en el médico. Las mujeres ponen énfasis en el cuidado de sí y de Dios:

\section{...sí, se cura si uno se cuida [...] yo no me cuido mucho, pero siempre estoy haciendo oración al Señor que me cuide, yo estoy rogando. ( $\mathrm{F}$, mujer, 65 años)}

Los varones también confían en Dios: "sí, confiando en Dios todopoderoso. Uno tiene que tener $\mathrm{fe}^{\prime \prime}$ ( $\mathrm{M}$, hombre, 54 años), pero los aspectos del cuidado tienen una presencia mucho más débil. En su discurso se distingue mejor una repetición de conceptos transmitidos por la iglesia evangélica, que podría definirse como un deber confiar.

Los resultados de las observaciones y entrevistas indican que la diabetes es una enfermedad, hasta hace poco, desconocida:

...no la conocía, en ese entonces muy poco se escuchaba de esa enfermedad, y ahora todo el mundo está enfermo de esa enfermedad. (M, hombre, 54 años)

Registramos dos casos de amputación en la comunidad, sin sobrevida. En el año 2016, el pastor de la comunidad tuvo una amputación y falleció al poco tiempo, y hace unos años atrás falleció una mujer a los pocos meses de la amputación. Como resultado de estas experiencias circula entre los varones el concepto de "muerte lenta" para referirse a la diabetes.

Observamos que se atribuyen a la diabetes muertes de padres y madres que por su descripción mejor se adecúan a muertes por Chagas:

...no sabían por qué morían, la alimentación, mi abuela ha fallecido hace más de veinte años de esa enfermedad. ( $E$, mujer, 48 años)

\begin{abstract}
Mi papá falleció cuando yo tenía seis años por esa enfermedad. Papá trabajaba en el cerco: cortaba leña y se mareó y se cayó. A la semana murió. (M, hombre, 54 años)
\end{abstract}

La aflicción de la actualidad de la diabetes, y sus recortes de sentido, parecen resignificar el dolor antiguo por las sucesivas pérdidas de los seres queridos, las tierras y las costumbres pasadas.

\section{Los servicios de salud: acceso, tratamiento e interculturalidad}

Las profesionales de salud del CAPS Misión Los Tapietes y del hospital de Tartagal reconocen la diversidad cultural de la población que se atiende en la zona. No obstante, no encontramos un dispositivo de salud intercultural funcionando en los servicios. En el CAPS se afirma que las y los pacientes:

...entienden bastante bien el castellano, ellos solos vienen a la atención. La secretaria es bilingüe. Está todo organizado: el agente sanitario hace el control antropométrico, búsqueda de tuberculosis asintomática, captación de embarazadas, peso y talla, y sintomatologías. Aquí se hace medicina de la misma forma que todos. (B, enfermera, CAPS)

En el barrio Tapiete la población está mezclada, al CAPS van personas de otros barrios, y la mayoría que asiste son mujeres. (G, médica clínica, CAPS)

...la población del hospital es de bajos recursos, preferentemente urbana. Se atienden indígenas, wichís, tapietes. (D, médica diabetóloga, hospital)

...la población que asiste al hospital es variada, criolla y aborigen: tobas, guaraníes, wichís. Pero los tobas y tapietes tienen mayor acceso, es más fácil la interpretación. (S, médica de familia, hospital) 
El relato refiere a que los tobas y tapietes hablan castellano, por lo cual la interacción es más accesible para la médica. Con base en nuestras observaciones, podemos afirmar que el estereotipo del indígena, al menos en el hospital, está más representado por el pueblo wichí, en cuya población hay un mayor uso de la lengua nativa y un mayor distanciamiento del sistema de salud pública. De modo que los efectores de salud reconocen que, entre los tapietes, la lengua no es un obstáculo en la atención, salvo en la gente mayor, pero aun así señalan diferencias culturales que no conducen a una relación necesariamente fluida con los servicios de salud.

Según la enfermera del CAPS, la diabetes irrumpió recientemente en la comunidad tapiete:

...hace solo tres años que los adultos llegan graves al hospital, y después los derivan al CAPS. No utilizan medicina tradicional porque no saben qué es esa enfermedad, van directamente al médico. (B, enfermera, CAPS)

En esta observación notamos un desconocimiento de las prácticas de atención cotidianas por parte de las y los pacientes. La médica clínica identifica la frecuencia de "hipertensión arterial y diabetes, sobrepeso y obesidad" entre pacientes del CAPS:

...las personas con diabetes tienen entre 45 y 60 años de edad, con una evolución de entre 10 y 15 años al momento de la presencia de los síntomas. [...] mayor concientización en las mujeres, aunque llegan descompensadas por las tareas domésticas, el cuidado de la familia. Los varones son más reactivos al diagnóstico. Hay predominio de mujeres porque consultan más. (G, médica clínica, CAPS)

Entre las causas de la enfermedad, la médica identifica "muy mala alimentación por las carencias: 'no puedo cocinar eso para mí, y a mis hijos ¿qué les hago?'”.

En el hospital, la médica de familia observa la presencia de diabetes, hipertensión arterial, artritis reumatoidea en población indígena y destaca menor presencia de obesidad y colesterolemia en población aborigen. Asimismo, señala que "es más de criollo que anda en moto o colectivo" tener estas enfermedades e identifica la presencia de diabetes después de los 50 años, por la alimentación, el cambio de hábitos y la falta de ejercicio. La médica diabetóloga afirma que:

...los pacientes indígenas Ilegan descompensados, con hiperglucemia. [Encuentra] "mayor seguimiento en las mujeres, más constancia y mayor adhesión al tratamiento. (S, médica de familia, hospital)

La emergencia de diabetes en la población indígena la explica en términos de:

...mayores hábitos de alimentación de
los nuestros: Coca-cola, urbanización,
ayuda social basada en harina, leche,
arroz, azúcar, alimentos más industriali-
zados, que antes no tenían. Los wichís y
los guaraníes ya no practican la agricul-
tura. (S, médica de familia, hospital)

De esta manera, las profesionales de la salud reconocen la emergencia de la diabetes y su presencia creciente en las comunidades indígenas. El predominio de mujeres con diagnóstico se explica en términos de acceso al sistema de salud de quienes, como madres, tienen la experiencia de asistir de forma más o menos regular a los CAPS para los controles y tratamiento de sus hijas e hijos; en suma, como parte de sus prácticas de cuidado en la familia.

En cuanto al tratamiento indicado por el sistema de salud pública, las profesionales de la salud señalaron:

[se realiza] una interconsulta con la nutricionista por obesidad, luego la médica indica la medicación: metformina, glibenclamida y, en tercer lugar, el control diario de presión arterial y mensual de peso en enfermería. Cada paciente tiene su cuaderno, donde lleva el control. Como no tenemos glucotest para moni- 
toreo, los médicos indican un laboratorio cada mes. (B, enfermera, CAPS)

...existen dificultades de acceso, puesto que tienen que hacer cola a las cuatro de la mañana. (G, médica clínica, CAPS)

...estamos tratando de vencer la barrera de venir, la cuestión de los turnos, para que no vengan a las cuatro de la mañana, implementar cuadernos de turnos. Llega un momento que abandonan todo. Trabajamos mejor con APS en comunidad, según los síntomas, con mayores de 45 años con antecedentes, y los agentes sanitarios conocen a las familias. (S, médica de familia, hospital)

...muchos toman medicina alternativa, al no estar interiorizados. A veces cuentan nombres de la zona y no los registro. (G, médica clínica, CAPS)

...cuando ya saben qué es lo que tienen, toman moringa, urucú para la presión, tusca como antibiótico para la herida, o aloe vera. (B, enfermera, CAPS)

...es más fácil que tomen la pastilla a que hagan la dieta y la actividad física. (S, médica de familia, hospital)

...existe un programa provincial que funciona en el hospital, pero es poco ágil. Los programas de distribución de medicamentos proveen de medicación mensual, tiras reactivas, glucómetro para pacientes con insulina, pero en el caso de los tapietes no hemos registrado insulinodependientes. [...] Se trabaja con la dieta recuperando lo que ellos comen junto con la nutricionista. Les recetamos metformina, glibenclamida, cremas, preparados de vaselina en la farmacia del hospital, estos son de acceso gratuito. Después de recibir el diagnóstico los pacientes toman yuyos. ( $\mathrm{D}$, médica diabetóloga, hospital)
De las observaciones y los relatos se deriva que existen dificultades de acceso a los servicios de salud y seguimiento de los tratamientos, vinculados muchas veces a las tensiones observadas entre los servicios y las comunidades. Estas tensiones se deben al distanciamiento de la biomedicina por parte de los pacientes indígenas, quienes además sienten que son regañados por los efectores de salud por su falta de adherencia al tratamiento.

Las complicaciones más frecuentes que la médica clínica observaba en el CAPS son retinopatía, necropatía diabética, lesiones. La enfermera afirmó que hubo amputaciones como las del pastor y otra mujer, y esto causó un grave impacto en la población. También indicaron que:

[hay un] señor que tiene una herida en la pierna, pero no quiere hacerse los estudios. Viene a hacerse curaciones y no se hace los estudios. Los hombres tienen más miedo de la aguja. (B, enfermera, CAPS)

...la complicación renal, sin diferencias de género. El pie diabético se deriva a Salta a curarse $u$ operarse, y ahí quedan internados. (S, médica de familia, hospital)

...todos Ilegan complicados, con glucemias altas, que se podrían haber detectado en los cinco años anteriores. Hay poca adherencia. Si andan bien, no los vuelvo a ver. Cada tres meses APS me envía pacientes complicados en su mayoría: cetoacidosis diabética, hiperglucemia, no recuerdo indígena amputado. (D, médica diabetóloga, hospital)

Entendemos que las complicaciones son frecuentes en la comunidad; sin embargo, es muy difícil sobrevivir a una amputación; de hecho, no hemos registrado casos que lo hayan logrado. Tampoco se indica insulina a las y los pacientes indígenas debido a las dificultades para el monitoreo de la glucemia en sangre en los hogares e incluso en el CAPS.

Hasta el año 2019, la presencia de programas de asistencia alimentaria en Misión Los Tapietes se limitaba a niñas y niños. 
Según la enfermera del CAPS, funcionaba un merendero municipal, un programa nacional para menores de seis años, además de las organizaciones de mujeres de la comunidad. En el hospital funcionaba un equipo de nutrición muy bien constituido.

[se siguen] planes de alimentación con las nutricionistas, se logran cambios, es impresionante cuando vamos a las casas. (S, médica de familia, hospital)

La médica diabetóloga afirma que hace un año, cuando ella misma renunció a la planta del hospital

[se discontinuaron] las charlas de alimentación, de concientización. Iban más mujeres porque eran al mediodía y los hombres no podían por el trabajo. El hombre sostiene la familia. No iban mujeres indígenas porque no les llegaba la información. (D, médica diabetóloga, hospital)

Si bien existe un subsidio de la Municipalidad de Tartagal para pacientes diabéticos, y asistencia social, en el caso de los tapietes, algunos reciben una pensión por discapacidad que les permite mantener a su familia.

Observamos que los programas de alimentación no se basan en los conocimientos y prácticas de la alimentación de la población indígena, y que suelen indicar el consumo de alimentos lácteos como yogur, que no solo no constituyen parte de la alimentación, sino que además son costosos y precisan refrigeración.

\section{DISCUSIÓN}

Las dimensiones trabajadas para describir los itinerarios terapéuticos de varones y mujeres con diabetes resultan coincidentes con la metodología y resultados de otros estudios fuera del ámbito argentino, en cuanto a la conceptualización de la diabetes, las causas de la enfermedad, las características del diagnóstico y del tratamiento. Mercado Martínez y Díaz
Medina ${ }^{(19)}$ revisaron 28 trabajos cualitativos etnográficos producidos en México sobre la epidemia de diabetes y encontraron estas dimensiones: experiencias y vivencias de las personas enfermas, trayectorias del padecimiento, y cómo atienden su padecimiento. García Serrano et al. ${ }^{(20)}$ generaron las siguientes categorías en un estudio en la comunidad Wixarika de México: conceptualización de la diabetes, terapéutica, génesis, y situación sociocultural de la diabetes.

Diversos trabajos indican cambios en los patrones de morbilidad y mortalidad caracterizada por una "transición epidemiológica" de carácter compleja. Es decir que, a los altos índices de enfermedades infecciosas, se sumaría el impacto de las enfermedades crónicas. Arnold et al. ${ }^{(21)}$ estudian las relaciones no exploradas entre diabetes y tuberculosis con el propósito de especificar las recomendaciones de la Organización Mundial de la Salud (OMS) sobre la interacción entre ambas condiciones. En Argentina, Fernández ${ }^{(22)}$ analiza el impacto de la diabetes, en tanto enfermedad emergente, en la dinámica de transmisión de la tuberculosis. Las interacciones observadas durante el trabajo de campo exploratorio -entre Chagas y diabetes, y entre diabetes y tuberculosis- pueden interpretarse desde los efectos de las condiciones de sindemia señaladas por Singer y $\mathrm{Scott}^{(23)}$. El concepto de sindemia implica el rol adverso e injusto de las condiciones sociales en la interacción de las enfermedades. La sindemia se desarrolla en situaciones de inequidad sanitaria, pobreza, violencia estructural. Implica una sinergia:

\footnotetext{
...la sindemia involucra la interacción adversa de enfermedades de todo tipo (ejemplo, infecciones, enfermedades crónicas no transmisibles, problemas de salud mental, exposición a tóxicos, desnutrición) y como resultado de tal interacción se produce un incremento en la carga de enfermedad en una población. ${ }^{(24)}$
}

Estas consideraciones imponen la reflexión sobre las nociones de la nosología de la biomedicina expresadas en la clasificación de 
enfermedades de la OMS y sus límites demarcatorios, o fronteras de la enfermedad, que operan en la base de la epidemiología clásica. Es preciso continuar investigando en esta línea para construir encuadres epistemológicos más complejos que eviten el reduccionismo y la simplificación de los análisis de la situación de salud de las poblaciones en contextos de subalternación y empobrecimiento.

En este marco, proponemos como hipótesis la existencia de líneas significativas de sentidos de continuidad retrospectiva entre la diabetes y el Chagas: se atribuyen a la diabetes muertes del pasado que por su descripción se adecúan más al Chagas. Diabetes y Chagas comparten los sentidos de la enfermedad silenciosa. Susan Sontag(25) había identificado una continuidad de sentidos entre la tuberculosis del siglo XIX y el cáncer del siglo XX, pero donde el cáncer conserva los sentidos antes atribuidos a la tuberculosis: lo no dicho, lo innombrable, "la enfermedad". Aquí parece haber un sentido inverso, retrospectivo, del presente hacia el pasado: se atribuye al Chagas del siglo XX los sentidos de la diabetes, emergente en el siglo XXI: la enfermedad silenciosa, una "muerte lenta", que se desencadena de manera súbita.

Smith-Morris ${ }^{(26)}$ indaga en la cronicidad de la diabetes entre los pima, de Nuevo México; su trabajo pregunta de qué manera la biotecnología moderna crea condiciones crónicas o identidades crónicas donde no existían antes. Encuentra que para los pima ser definidos como diabéticos se vincula a una experiencia histórica y política determinada, la historia de colonización, pérdida de territorio, dependencia de los commodities del gobierno y el control sobre sus comunidades. Estroff ${ }^{(27)}$ indica que la cronicidad consiste en la fusión de la identidad con el diagnóstico, una limitación de los roles sociales e identidades que lleva de ser paciente a discapacitado, una desmoralización que implica pasar de ser una persona que tiene una enfermedad a ser una persona que es una enfermedad o diagnóstico. La noción de cronicidad constituye un proceso de identificación con la enfermedad que se tiene, un movimiento de tener una enfermedad a ser una persona habitada por la enfermedad ${ }^{(26)}$. No hemos encontrado sentidos asociados con la enfermedad crónica entre las personas con diabetes entrevistadas en Misión Los Tapietes. Hemos observado y registrado expectativas de curación de la diabetes a través de diversas estrategias. Estas observaciones requieren ser profundizadas en futuros trabajos para rendir cuenta de la resistencia social y cultural que pueden estar expresando, como también para analizar, desde la perspectiva de género, las diferencias entre varones y mujeres en relación con el diagnóstico de diabetes y los tratamientos biomédicos. Consideramos que tanto en varones como en mujeres, con particularidades propias de los procesos diferenciales y complementarios de subjetivación generacionales de la comunidad tapiete, estos mecanismos se vinculan con líneas de fuga en relación con las prescripciones médicas. Como explica Fernández ${ }^{(28)}$, en Argentina, los cuerpos privados, humillados, devastados por el hambre, el sobretrabajo o la desocupación, sujetos del estigma, el maltrato y la exclusión, han obedecido, han acatado, pero también han resistido a las imposiciones sociales. En el marco de relaciones interétnicas de subalternidad en relación con la sociedad nacional y los procesos históricos de despojo de la tierra, de la lengua y de los saberes y las prácticas tradicionales, las mujeres y los varones tapietes entrevistados atravesaron una relación refractaria con la biomedicina. Desde esas posiciones de género podrían estar resistiendo de manera diferencial, a través de la negación o de la confrontación, a las prescripciones médicas, entendidas como normalizadoras de la incorporación de un orden social, que es vivenciado en tanto ajeno a sus conceptualizaciones de la salud y enfermedad.

Diversos estudios indican que los pacientes diabéticos relacionan las causas de su padecer con algún trauma y cómo el manejo de la vida emocional es para ellos importante para controlar la enfermedad. Montesi ${ }^{(29)}$ señala que para el caso de los indígenas ikojts de México hay una conexión entre diabetes y emociones fuertes y negativas. Estudios cualitativos realizados en México encontraron 
pensamientos y sentimientos similares en varones y mujeres en relación con las condiciones biomédicas de la diabetes ${ }^{(2)}$, pero diferencias en los procesos de subjetivación de género: para los varones es una enfermedad que los vulnera y se convierte en reto, para las mujeres es un castigo. En las mujeres de sectores populares, la diabetes está relacionada con sentimientos negativos de rabia y tristeza, y se la vincula a la pérdida de placer en el plano alimentario. Las diferencias de género del significado de la enfermedad se reflejan en las prácticas de cuidado que ponen en riesgo la vida ${ }^{(3,30)}$. Torres-López et al. ${ }^{(31)}$ encuentran en Guadalajara que la diabetes es un padecimiento con connotaciones femeninas, que se representa también por "tener azúcar" en la sangre. Nuestras observaciones preliminares en las formas de nombrar y significar la enfermedad merecen ser profundizadas desde la perspectiva de género: especialmente las asociaciones entre diabetes y azúcar, y los sentimientos encontrados ante la condición de "enfermo" o "paciente".

En otro plano, la ausencia del sentido de cronicidad y la perspectiva de curación de la diabetes invitan a repensar las prácticas de atención de los servicios públicos de salud. Estudios previos en comunidades indígenas de Argentina indican que la gente mayor tiene las mayores dificultades con la comprensión y el acercamiento a los efectores de salud por las diferencias lingüísticas y culturales ${ }^{(32)}$. Entre los wixárica de México se encontró que el proceso de comprensión, explicación y aceptación de la condición de diabetes resulta confrontativo y doloroso e insume varios meses para la instrumentación de acciones de cuidado de la salud ${ }^{(33)}$. No hemos encontrado en el CAPS de Misión Los Tapietes ni en el hospital de Tartagal dispositivos de salud intercultural, pero sí dificultades de acceso al diagnóstico oportuno y a la continuidad de los tratamientos de las mujeres y los varones con diabetes. Es preciso flexibilizar las categorías de la biomedicina, incorporando visiones y valores locales, si se pretende ejercer el cuidado de la salud en contextos interculturales. La incorporación de agentes sanitarios residentes en las comunidades a la atención primaria de salud, y de enfermeros/as y médicos/as indígenas en los CAPS parece ser la mejor iniciativa concreta para una articulación entre el Estado y las comunidades en el cuidado y la atención del proceso de salud y enfermedad. Es preciso trabajar en su inserción social y cultural para el fortalecimiento de las estrategias de salud intercultural que articulen el reconocimiento de los saberes y las prácticas tradicionales de alimentación, los cuidados integrales de la salud y una perspectiva de derechos identitarios que colabore con la recuperación de la salud y el bienestar de las comunidades indígenas. Esta iniciativa no podría implementarse por fuera del mejoramiento de las condiciones de existencia de la población destinataria ${ }^{(34)}$ y de la articulación entre las reivindicaciones territoriales de las poblaciones indígenas con el derecho a la salud y la alimentación. El acceso a la tierra y al agua, que permita la provisión de alimentos frescos y nutritivos, y contribuya a disminuir el consumo de bebidas azucaradas y alimentos procesados, resultan condiciones necesarias para la implementación de estrategias interculturales en la atención primaria de salud.

La determinación social de la salud, enfoque propio de la epidemiología crítica latinoamericana $^{(35)}$ resulta un modelo explicativo de la complejidad de la situación epidemiológica de las comunidades, puesto que articula los modos de vivir, de enfermar y de morir con las formas de reproducción social y permite analizar los diferenciales de salud de acuerdo con la matriz de triple inequidad -de etnia, de clase y de género- estructural en las sociedades latinoamericanas. La producción y reproducción de desigualdades sobre las comunidades periurbanas como Misión Los Tapietes y su historia de explotación laboral en los ingenios, los desplazamientos y desalojos reiterados, las pérdidas territoriales y los loteos de las comunidades, las condiciones de hacinamiento, las desvalorizaciones constantes de la diversidad cultural vienen desarticulando las formas propias de reproducción social, las prácticas de producción agrícola, de alimentación tradicional, de medicina antigua, de fiestas y rituales. Como consecuencia, y sumado 
a la crisis social y económica que vive la sociedad nacional, se produce un impacto en los cuerpos, que enferman, y las subjetividades, que deprimen. Una vez más se confirma la inviabilidad del modo de producción del capital para la salud y la naturaleza.

La Reforma Constitucional de 1994, la Ley 23302 de Comunidades Indígenas, el Convenio 169 de la $\mathrm{OIT}^{(36)}$ y otras normativas vigentes abogan por el respeto a la diversidad étnica y el derecho a la salud de los pueblos originarios, así como al ejercicio de la medicina ancestral. Sin embargo, el respeto de sus derechos está muy lejos de los estándares aceptables a nivel internacional. La salud está relacionada también con el ambiente, el acceso a la tierra, la integridad cultural, las relaciones intersubjetivas y comunitarias. ¿Pero cuáles son las oportunidades para ejercer el derecho a la salud si las comunidades están permanentemente sometidas a la presión sobre las tierras, los cuerpos y los imaginarios?

\section{AGRADECIMIENTOS}

A las y los habitantes de Misión Los Tapietes, Tartagal, provincia de Salta; a las profesionales de salud del Centro de Atención Primaria de Salud de Misión Los Tapietes y del Hospital "Juan Domingo Perón" de Tartagal.

\section{REFERENCIAS BIBLIOGRÁFICAS}

1. Cuyul A, Rovetto $M$, Specogna M. Pueblos indígenas y sistemas de información en salud: La variable étnica en seis provincias argentinas. Revista Argentina de Salud Pública. 2011;2(7):12-18.

2. Reyes Luna AG, Salguero Velázquez A, Tena Guerrero O. Diagnóstico de Diabetes Mellitus tipo II: significado y diferencias de género en hombres y mujeres. Alternativas en Psicología. 2015;18(32):125-144.

3. Salcedo-Rocha AL, García de Alba-García JE, FrayreTorres MJ. López-Coutino, B. Género y control de diabetes mellitus 2 en pacientes del primer nivel de atención. Revista Médica del Instituto Mexicano del Seguro Social. 2008;46(1):73-81.

4. Concha Toro M, Rodríguez Garcés CR. Funcionalidad familiar en pacientes diabéticos e hipertensos compensados y descompensados. Theoria, 2010;19:41-50.

5. Trujillo Olivera LE, Nazar Beutelspacher A. Autocuidado de diabetes: una mirada con perspectiva de género. Estudios Demográficos y Urbanos. 2011;26(3):639-670. doi: 10.24201/edu.v26i3.1377.

6. Ferzacca S. Diabetes and Culture. Annual Review of Anthropology. 2012;41:411-426. doi: 10.1146/annurevanthro-081309-145806.

7. Ferreira ML, Lang GC. Indigenous Peoples and Diabetes. Community Empowerment and Wellness. Durham: Carolina Academic Press; 2006.

8. González HA, Hirsch S, Ciccone F, Montani C. Ecos del mundo vegetal entre los Tapietes de Argentina. München: Lincom; 2017.
9. Brave Heart MYH, De Bruyn LM. The American Indian holocaust: Healing historical unresolved grief. American Indian and Alaska Native Mental Health Research. 1998;8(2);60-82.

10. Korn LE, Ryser RC. Burying the Umbilicus: Nutrition Trauma, Diabetes and Traditional Medicine in Rural West Mexico. En: Ferreira ML, Lang GC. Indigenous Peoples and Diabetes. Community Empowerment and Wellness. Durham: Carolina Academic Press; 2006. p. 231-277.

11. Korn LE. Community trauma and development. Fourth World Journal. 2003;5(1):1-9.

12. Menéndez EL. De saberes médicos, populares y científicos. Buenos Aires: Lugar Editorial; 2018.

13. Menéndez EL. Salud intercultural: propuestas, acciones y fracasos. Ciência \& Saúde Coletiva. 2016;21(1): 109-118.

14. Conrad P, Baker K. The Social construction of illness: Key insights and policy implications. Journal of Health and Social Behavior. 2010;51(Supl):S67-S79. doi: 10.1177/ 0022146510383495 .

15. Menéndez EL. Interculturalidad, diferencias y antropología at home: Algunas cuestiones metodológicas. En: Fernández Juárez G. (coord.). Salud e interculturalidad en América Latina: Antropología de la salud y crítica intercultural. Quito: Ediciones Abya-Yala, 2006. p. 51-66.

16. Aguirre P. Una historia social de la comida. Buenos Aires: Lugar Editorial; 2017.

17. Menéndez EL. Antropologia médica e epidemiologia. En: Almeida Filho N, Barreto ML, Veras RP, Barata RB, (orgs.). Teoria epidemiológica hoje: fundamentos, interfaces, tendências. Rio de Janeiro: Fiocruz; 1998. p. 81-103.

18. Minayo MCS. La artesanía de la investigación cualitativa. Buenos Aires: Lugar Editorial; 2013.

19. Mercado Martínez FJ, Díaz Medina BA. Vivir y morir de diabetes en México ¿qué piensan y qué hacen las personas que la padecen? En: Chapela MC, (ed.). En el debate: diabetes en México. México: Universidad Autónoma Metropolitana-Xochimilco; 2010. p. 111-134. 
20. García Serrano V, Crocker R, García de Alba J. Creencias sobre la diabetes mellitus tipo 2 de la etnia Wixarika del occidente de México. Revista Chilena de Antropología. 2017;35:113-130.

21. Arnold Y, Licea M, Castelo L. Diabetes mellitus y tuberculosis. Revista Peruana de Epidemiología. 2012;16(2): 76-83.

22. Fernández HR. Impacto de la diabetes en la dinámica de transmisión de la tuberculosis. [Tesis de Maestría]. Brasil: Fiocruz; 2012.

23. Singer MC, Scott C. Syndemics and public health: Reconceptualizing disease in bio-social context. Medical Anthropology Quarterly. 2003;17(4):423-441. doi: 10.1525/maq.2003.17.4.423.

24. Singer M, Bulled N, Ostrach B. Syndemics and human health: Implications for prevention and intervention. Annals of Applied Anthropology. 2012;36(2):205-211. doi: 10.1111/napa.12000.

25. Sontag S. La enfermedad y sus metáforas: El sida y sus metáforas. Buenos Aires: Taurus; 2003.

26. Smith-Morris $\mathrm{C}$. The chronicity of life, the acuteness of diagnosis. En: Manderon L, Smith-Morris C, (eds.). Chronic conditions, fluid states: Chronicity and the anthropology of illness. New Brunswick: Rutgers University Press; 2010. p. 21-37.

27. Estroff SE. Identity, disability and schizophrenia: The problem of Chronicity. En: Lindenbaum S, Lock M, (eds.). Knowledge, power and practice: The anthropology of Medicine and everyday life. Berkeley: University of California Press; 1995. p. 247-287.

28. Fernández AM. Las lógicas colectivas: Imaginarios, cuerpos y multiplicidades. Buenos Aires: Biblos; 2008.

29. Montesi L. "Como si nada": Enduring violence and diabetes among women in Southern Mexico. Medi- cal Anthropology. 2018;37(3):206-220. doi: 10.1080/ 01459740.2017 .1313253 .

30. Trujillo Olivera LE, Nazar Beutelspacher A. Autocuidado de diabetes: una mirada con perspectiva de género. Estudios Demográficos y Urbanos. 2011;26(3): 639-670.

31. Torres-López TM, Sandoval-Díaz M, Pando-Moreno M. Sangre y azúcar: representaciones sobre la diabetes de los enfermos crónicos en un barrio de Guadalajara, México. Cadernos de Saúde Pública. 2005;21(1):101-110.

32. Cebolla Badie M, Hirsch S, Dell'Arciprete A, Orlando MF. Los itinerarios terapéuticos y la importancia de las terapias con medicamentos industriales en comunidades pilagá, qom, guaraní y mbya-guaraní de Argentina. En: Hirsch S, Lorenzetti M, Salomón OD, (comps.). Procesos de investigación e intervención en salud en comunidades indígenas de la Argentina. Posadas: INMET; 2015.

33. Quiñonez-Tapia F, Vargas-Garduño ML, SolteroAvelar R. Los wixáritari con diabetes mellitus y sus vínculos con la enfermedad: desde la aparición del síntoma hasta una primera explicación. Salud Colectiva. 2019;15:e1856. doi: 10.18294/sc.2019.1856.

34. Lorenzetti M. Relaciones interétnicas y prácticas de atención de la salud en el Chaco Salteño. Corpus Archivos Virtuales de la Alteridad Americana. 2011;1(2):1-18.

35. Breilh J. La determinación social de la salud como herramienta de transformación hacia una nueva salud pública (salud colectiva). Revista Facultad Nacional de Salud Pública. 2013;31(supl 1):S13-S27.

36. Consejos de Organización Aborigen de Jujuy. Experiencias en la recuperación de plantas medicinales del pueblo guaraní [Internet]. San Salvador: COAJ, Welfriedensdienst (citado 12 sep 2019). Disponible en: https:// tinyurl.com/y22lqpjh. 